\title{
Generalized gluing for Einstein constraint equations
}

\author{
Lorenzo Mazzieri
}

Received: 19 December 2007 / Accepted: 5 May 2008 / Published online: 7 June 2008

(C) The Author(s) 2008

\begin{abstract}
In this paper we construct a family of new (topologically distinct) solutions to the Einstein constraint equations by performing the generalized connected sum (or fiber sum) of two known compact $m$-dimensional constant mean curvature solutions $\left(M_{1}, g_{1}, \Pi_{1}\right)$ and $\left(M_{2}, g_{2}, \Pi_{2}\right)$ along a common isometrically embedded $k$-dimensional sub-manifold $\left(K, g_{K}\right)$. Away from the gluing locus the metric and the second fundamental form of the new solutions can be chosen as close as desired to the ones of the original solutions. The proof is essentially based on the conformal method and the geometric construction produces a polyneck between $M_{1}$ and $M_{2}$ whose metric is modeled fiber-wise (i. e. along the slices of the normal fiber bundle of $K$ ) around a Schwarzschild metric; for these reasons the codimension $n:=m-k$ of $K$ in $M_{1}$ and $M_{2}$ is required to be $\geq 3$. In this sense our result is a generalization of the Isenberg-Mazzeo-Pollack gluing, which works for connected sum at points and in dimension 3. The solutions we obtain for the Einstein constraint equations can be used to produce new short time vacuum solutions of the Einstein system on a Lorentzian $(m+1)$-dimensional manifold, as guaranteed by a well known result of Choquet-Bruhat.
\end{abstract}

Mathematics Subject Classification (2000) $\quad 53 \mathrm{C} 21 \cdot 58 \mathrm{~J} 60 \cdot 83 \mathrm{C} 05 \cdot 53 \mathrm{~A} 30 \cdot 57 \mathrm{R} 65$

\section{Introduction and statement of the result}

\subsection{CMC solutions and conformal method}

It is well known [4] that short time vacuum solutions for the Einstein hyperbolic system on a Lorentzian $(m+1)$-dimensional manifold $(Z, \gamma)$ may be obtained from solutions of

\footnotetext{
L. Mazzieri

Scuola Normale Superiore, Piazza dei Cavalieri 7, 56126 Pisa, Italy

e-mail: 1.mazzieri@sns.it

L. Mazzieri $(\bowtie)$

Max-Plank-Instutut für Gravtationsphysik Albert-Einstein-Institut, Am Mühlenberg 1, 14476 Golm, Germany

e-mail: lorenzo.mazzieri@aei.mpg.de
} 
the Einstein constraint equations on a $m$-dimensional space-like Riemannian submanifold $(M, g)$ of $Z$. In fact the solutions to the constraints form a suitable set of vacuum initial data for the hyperbolic Cauchy problem (for further details see [2]). More precisely, when we are talking about a solution of the constraints we refer to a triple $(M, g, \Pi)$, where $M$ is a smooth manifold and $g$ and $\Pi$ are symmetric $(2,0)$ tensors (respectively the induced Riemannian metric and the second fundamental form), verifying the relationships

$$
\begin{aligned}
\operatorname{div}_{g} \Pi-d\left(\operatorname{tr}_{g} \Pi\right) & =0 \\
R_{g}-|\Pi|_{g}^{2}+\left(\operatorname{tr}_{g} \Pi\right)^{2} & =0,
\end{aligned}
$$

where $\operatorname{div}_{g}$ and $\operatorname{tr}_{g}$ are respectively the divergence operator and the trace operator computed with respect to the metric $g$ and $R_{g}$ is the scalar curvature of the metric $g$.

In the case we are looking for constant mean curvature (CMC) solutions of the constraints (i.e. when $\tau:=\operatorname{tr}_{g} \Pi$ is a constant) the system above becomes equivalent to an uncoupled system by means of the so called conformal method. Following [4], [11] and [10], one can split the second fundamental form $\Pi$ into trace free and pure trace parts

$$
\Pi=\mu+\frac{\tau}{m} g,
$$

where $\mu$ is a symmetric 2-tensor such that $\operatorname{tr}_{g} \mu=0$.

Then it is convenient to consider the double conformal change

$$
\begin{aligned}
& g=u^{\frac{4}{m-2}} \bar{g} \\
& \mu=u^{-2} \bar{\mu},
\end{aligned}
$$

where the conformal factor $u$ is a positive smooth function on $M$.

It is now straightforward to see that $g$ and $\Pi$ verify the Einstein constraint equations (1) and (2) if and only if the following holds for $\bar{g}, \bar{\mu}$ and $u$

$$
\begin{aligned}
\operatorname{tr}_{\bar{g}} \bar{\mu} & =0 \\
\operatorname{div}_{\bar{g}} \bar{\mu} & =0 \\
\operatorname{Lic}_{\bar{g}}(u) & =0,
\end{aligned}
$$

where Lic is the semi-linear elliptic operator given by

$$
\operatorname{Lic}_{\bar{g}}(u)=\Delta_{\bar{g}} u+c_{m} R_{\bar{g}} u-c_{m}|\bar{\mu}|_{\bar{g}}^{2} u^{-\frac{3 m-2}{m-2}}+c_{m} \frac{m-1}{m} \tau^{2} u^{\frac{m+2}{m-2}}
$$

with $c_{m}=-(m-2) /[4(m-1)]$ (also notice that our Laplacian is negative definite).

Therefore, if we start with a metric $\bar{g}$ and a real number $\tau$, in order to produce a $\tau$-CMC solution for the Einstein constraints it is sufficient to provide a symmetric $\bar{g}$-transverse (7) $\bar{g}$-traceless (6) tensor (briefly TT-tensor) and the right conformal factor, it is to say a solution of the Lichnerowicz equation (8).

In this context and because of their physical meaning [2], we will refer in the following to the Eq. (1) [or equivalently to the Eq. (7)] as the momentum constraint and to the Eq. (2) [or equivalently to the Eq. (8)] as the Hamiltonian or energy constraint.

\subsection{Strategy of the gluing and statement of the main result}

In the spirit of [10] suppose now that we start with two Cauchy data sets, namely two solutions $\left(M_{i}, g_{i}, \mu_{i}, u_{i}, \tau\right), i=1,2$ to Eqs. (6)-(8) (notice that this corresponds, modulo 
the conformal changes $\tilde{g}_{i}=u_{i}^{4 /(m-2)} g_{i}$ and $\tilde{\mu}_{i}=u_{i}^{-2} \mu_{i}$, to considering two sets of $\tau$-CMC solutions $\left(M_{i}, \tilde{g}_{i}, \tilde{\Pi}_{i}=\tilde{\mu}_{i}+(\tau / m) \tilde{g}_{i}\right), i=1,2$ to Eqs. (1), and (2), and suppose that we construct the generalized connected sum of the compact $m$-dimensional manifolds $M_{1}$ and $M_{2}$ along a common isometrically embedded $k$-dimensional Riemannian submanifold $\left(K, g_{K}\right)$. This construction consists in excising a small $\varepsilon$-tubular neighborhood (i.e. a tubular neighborhood of size $\varepsilon \in(0,1))$ of $K$ in both the starting manifolds and in identifying the differentiable structures along the leftover boundaries as explained in [19] and summarized in section 2. The purpose is then to endow-in correspondence to each value of $\varepsilon$ - the new manifold $M_{\varepsilon}=M_{1} \sharp_{K, \varepsilon} M_{2}$ with a Riemannian structure $g_{\varepsilon}$ and a symmetric TT-tensor $\mu_{\varepsilon}$ such that a solution $u_{\varepsilon}$ to Eq. (8) can be found, with the same $\tau$ as the starting Cauchy data sets.

As is typical of the gluing results, the new solution has to preserve the information about the starting solutions insofar as is possible. In our case the metric $g_{\varepsilon}$ will coincide by construction with the metrics $g_{1}$ and $g_{2}$ away from the gluing locus. Moreover, as the geometric parameter $\varepsilon$ tends to zero, the metric $g_{\varepsilon}$ tends to the metric $g_{i}$ and the TT-tensor $\mu_{\varepsilon}$ tends to $\mu_{i}$ on the compact sets of $M_{i} \backslash K$, with respect to the $\mathcal{C}^{2}$ topology, for $i=1,2$. In addition, we can make the conformal factor $u_{\varepsilon}$ as close to the constant one as we want, by choosing $\varepsilon$ to be small. In this sense, we are allowed to look at the metric $g_{\varepsilon}$ and at the TT-tensor $\mu_{\varepsilon}$ as an approximate solution of the system (1)-(2), which can be made exact by means of a small conformal perturbation $u_{\varepsilon} \simeq 1$

$$
\begin{aligned}
\tilde{g}_{\varepsilon} & =u_{\varepsilon}^{\frac{4}{m-2}} g_{\varepsilon}, \\
\tilde{\Pi}_{\varepsilon} & =u_{\varepsilon}^{-2} \mu_{\varepsilon}+\frac{\tau}{m} u_{\varepsilon}^{\frac{4}{m-2}} g_{\varepsilon} .
\end{aligned}
$$

As already explained, the real advantage in considering CMC solutions is that one has an uncoupled system (6)-(8) to solve instead of the system (1)-(5). In particular, once an approximate solution metric $g_{\varepsilon}$ is available, the natural way to proceed is to solve first the equations (6) and (7), and then to put the solution $\mu_{\varepsilon}$ in the Eq. (8) and solve this one for $u_{\varepsilon}$. Since the latter equation is nonlinear and we wish to solve it by means of a perturbation argument which allows us to obtain a new solution which is as close as we want to the starting ones when $\varepsilon$ tends to zero (notice that this corresponds to $u_{\varepsilon} \rightarrow 1$ as $\varepsilon \rightarrow 0$ ), we are led to linearize the Lichnerowicz operator around the constant one and to consider the leftover terms as error terms. Among these error terms, the squared norm of $\mu_{\varepsilon}$ plays a significant role.

As a consequence of this special role of $\mu_{\varepsilon}$, it is crucial to get an $\varepsilon$-uniform bound for solutions of the Eq. (7). In this form the momentum constraint is a linear system of partial differential equations and there is a standard two step procedure to produce trace free solutions of it [21]. In our case we will proceed as follows. Starting with $\mu_{1}$ and $\mu_{2}$ and using suitable cut-off functions, we produce a $g_{\varepsilon}$-trace free symmetric 2-tensor $\mu$, which in general is not a solution to (7) (notice that $\mu$ actually depends on $\varepsilon$ as long as it has to be trace free with respect to the metric $g_{\varepsilon}$ ). The second step consists then in finding a correction term $\sigma_{\varepsilon}$ which repairs the momentum constraint (i.e., $\operatorname{div}_{g_{\varepsilon}}\left(\mu+\sigma_{\varepsilon}\right)=0$ ). Since the system is largely underdetermined, we may force the solution to have a special shape. In particular we look for a solution of the form $\sigma_{\varepsilon}=D_{g_{\varepsilon}} X$, where $X$ is a vector field on $M$ and $D_{g_{\varepsilon}}$ is the so called conformal Killing operator for the metric $g_{\varepsilon}$. The conformal Killing operator acts in general as a map from vector fields to symmetric trace free 2-tensors and for an arbitrary metric $g$ is defined as 


$$
D_{g} X=\frac{1}{2} \mathcal{L}_{X} g-\frac{1}{m}\left(\operatorname{div}_{g} X\right) \cdot g
$$

where $\mathcal{L}$ is the Lie derivative. This operator enjoys a nice algebraic property: it is the negative of the formal adjoint of the divergence applied to symmetric trace free 2-tensors. More precisely

$$
D_{g}=-\left(\sharp \operatorname{div}_{g}\right)^{*} .
$$

As a consequence, when we perform the repair of the momentum constraint, we are induced to consider the elliptic self-adjoint operator $L_{g}:=-\sharp \operatorname{div}_{g} \circ D_{g}=D_{g}^{*} \circ D_{g}$, known as the vector Laplacian, and to solve the equation

$$
L_{g} X=\sharp \operatorname{div}_{g} \mu
$$

with respect to each $g_{\varepsilon}$ metric, therefore providing the solutions with an a priori $\varepsilon$-uniform bound.

The vector fields in the kernel of $D_{g}$ are called conformal Killing vector fields. In fact their flow leaves the metric invariant up to conformal changes; in other words they preserve the conformal class of the metric. For technical reasons, in order to deduce the $\varepsilon$-uniform estimate, we have to require a non-degeneracy assumption about the conformal Killing vector fields of the starting manifolds. The hypothesis we need is the following:

Non-degeneracy condition. There are no nontrivial conformal Killing vector fields on either $\left(M_{1}, g_{1}\right)$ or $\left(M_{2}, g_{2}\right)$.

Notice that because of the different geometric construction this assumption is slightly different from the non-degeneracy condition required in [10]. In fact the IMP gluing works under the assumption that there are no nontrivial conformal Killing vector fields on $\left(M_{1}, g_{1}\right)$ and $\left(M_{2}, g_{2}\right)$ wich vanish at the excised points.

In analogy with [10] we also need the assumption that both $\tilde{\Pi}_{1}$ and $\tilde{\Pi}_{2}$ are non identically zero. This guarantees, via the maximum principle, the injectivity of the linearized Lichnerowicz operators of the metrics $g_{i}$ around the constant one, namely

$$
\Delta_{g_{i}}-\left|\mu_{i}\right|_{g_{i}}^{2}-\tau^{2} / m, \quad i=1,2 .
$$

Notice that in the case where $\tilde{\Pi}_{1} \equiv 0 \equiv \tilde{\Pi}_{2}$ the Einstein constraint equations reduces to $R_{g_{1}}=0$ and $R_{g_{2}}=0$. In this situation the starting Cauchy data sets are said to be time symmetric and our problem reduces to constructing a scalar flat metric on the generalized connected sum of two scalar flat Riemannian manifolds. This can be done if both $\left(M_{1}, g_{1}\right)$ and $\left(M_{2}, g_{2}\right)$ are non Ricci flat, as shown in [18].

Following the strategy summarized above, we can prove the following result

Theorem 1 Let $\left(M_{1}, \tilde{g}_{1}, \tilde{\Pi}_{1}\right)$ and $\left(M_{2}, \tilde{g}_{2}, \tilde{\Pi}_{2}\right)$ be two compact $m$-dimensional $C M C$ solutions to the Einstein constraint equations (1)-(2) having the same constant mean curvature $\tau$ and verifying the non-degeneracy condition. Moreover suppose that both $\tilde{\Pi}_{1}$ and $\tilde{\Pi}_{2}$ are non identically zero and let $\left(K, \tilde{g}_{K}\right)$ be a common isometrically embedded $k$-dimensional sub-manifold with codimension $n:=m-k \geq 3$ such that the normal bundles of $K$ in $M_{1}$ and in $M_{2}$ are diffeomorphic. Then there exists a real value $\varepsilon_{0} \in(0,1)$ such that for every $\varepsilon \in\left(0, \varepsilon_{0}\right)$ it is possible to endow the $\varepsilon$-generalized connected sum $M_{\varepsilon}=M_{1} \sharp_{K, \varepsilon} M_{2}$ of $M_{1}$ and $M_{2}$ along $K$ with a metric $\tilde{g}_{\varepsilon}$ and a second fundamental form $\tilde{\Pi}_{\varepsilon}$ such that the triple $\left(M_{\varepsilon}, \tilde{g}_{\varepsilon}, \tilde{\Pi}_{\varepsilon}\right)$ is still a $\tau-C M C$ solution to the Einstein constraint equations.

Moreover the new metric $\tilde{g}_{\varepsilon}$ and the new symmetric TT-tensor $\tilde{\mu}_{\varepsilon}$ tend to the starting metric $\tilde{g}_{i}$ and to the starting symmetric TT-tensor $\tilde{\mu}_{i}$ respectively in the $\mathcal{C}^{2}$ topology on the compact sets of $M_{i} \backslash K$, for $i=1,2$, as the geometric parameter $\varepsilon$ tends to zero. 


\section{The geometric construction}

The aim of this section is to give a precise description of the generalized connected sum and to present a way to construct a family of approximate solution metrics $\left(g_{\varepsilon}\right)_{\varepsilon \in(0,1)}$; these are metrics which, when $\varepsilon$ varies in a sufficiently small range, can be perturbed to the final metric $\tilde{g}_{\varepsilon}$ by means of a small (i.e. close to one) conformal factor $u_{\varepsilon}$, this one being a solution to the Lichnerowicz equation with respect to the metric $g_{\varepsilon}$, the constant mean curvature $\tau$ and a suitable TT-tensor $\mu_{\varepsilon}$. At the end of this section we also present the construction of a symmetric $g_{\varepsilon}$-trace free tensor $\mu=\mu(\varepsilon)$ by means of a warped cut-off method (then, repairing this $\mu$ by means of a suitable symmetric tensor $\sigma_{\varepsilon}$, we will find the TT-tensor $\mu_{\varepsilon}:=\mu+\sigma_{\varepsilon}$ mentioned above).

The construction we present here is the same as [19]. Nevertheless, in order to make the exposition self contained and to fix the notation, we recall it here. The reason why this construction yields a good ansatz relies on the fact that the hamiltonian constraint is very similar to the Yamabe equation treated in [19], and since we want to produce analogous results (i.e. a conformal factor very close to one), we choose to solve our equation using the analytical tools and the geometric construction which have been successful with the Yamabe problem of [19].

Let $\left(K, g_{K}\right)$ be a $k$-dimensional Riemannian manifold isometrically embedded in both the $m$-dimensional Riemannian manifolds $\left(M_{1}, g_{1}\right)$ and $\left(M_{2}, g_{2}\right)$, we label the embedding maps as follows

$$
\iota_{i}: K \hookrightarrow M_{i}
$$

We assume that the isometric map $\iota_{1}^{-1} \circ \iota_{2}: \iota_{1}(K) \rightarrow \iota_{2}(K)$ extends to a diffeomorphism between the normal bundles of $\iota_{i}(K)$ in $\left(M_{i}, g_{i}\right), i=1,2$. To simplify the notations and the computations, here and in the following the injectivity radius of $K$ in both the manifolds is supposed to be greater than one, so that we are allowed to manipulate the differential and the metric structure on a fixed tubular neighborhood of $K$ in $M_{1}$ and $M_{2}$ of size one (gluing locus). This construction can obviously be modified in order to make the gluing locus as small as desired, and in particular smaller than the injectivity radii. For a fixed $\varepsilon \in(0,1)$, we describe the construction of the generalized connected sum (or fiber sum) of $M_{1}$ and $M_{2}$ along $K$ and the definition of the metric $g_{\varepsilon}$ in local coordinates. The fact that this construction yields a globally defined metric will follow at once.

Let $U^{k}$ be an open set of $\mathbb{R}_{z}^{k}, B^{n}$ the $n$-dimensional open ball of radius one in $\mathbb{R}_{x}^{n}$, where $n:=m-k \geq 3$ is the codimension of $K$. For $i=1,2, F_{i}: U^{k} \times B^{n} \rightarrow W_{i} \subset M_{i}$ given by

$$
F_{i}(z, x):=\exp _{(z, 0)}^{M_{i}}(x),
$$

defines local Fermi coordinates near the coordinate patches $F_{i}(\cdot, 0)(U) \subset \iota_{i}(K) \subset M_{i}$. In these coordinates, the metric $g_{i}$ can be decomposed as

$$
g_{(i)}(z, x)=g_{j l}^{(i)} d z^{j} \otimes d z^{l}+g_{\alpha \beta}^{(i)} d x^{\alpha} \otimes d x^{\beta}+g_{j \alpha}^{(i)} d z^{j} \otimes d x^{\alpha},
$$

and it is well known that in this coordinate system

$$
g_{\alpha \beta}^{(i)}=\delta_{\alpha \beta}+\mathcal{O}\left(|x|^{2}\right) \quad \text { and } \quad g_{j \alpha}^{(i)}=\mathcal{O}(|x|) .
$$

In order to perform the identification between $W_{1}$ and $W_{2}$ and in order to glue the metrics together and define $g_{\varepsilon}$, we partially change the coordinate system, by setting $x=\varepsilon \mathrm{e}^{-t} \theta$ on $F_{1}^{-1}\left(W_{1}\right)$ and $x=\varepsilon \mathrm{e}^{t} \theta$ on $F_{2}^{-1}\left(W_{2}\right)$, for $\varepsilon \in(0,1), \log \varepsilon<t<-\log \varepsilon, \theta \in S^{m-k-1}$. 
Actually, we will modify the starting metrics only on the hollow domain $U^{k} \times A^{n}\left(\varepsilon^{2}, 1\right)$ (where $A^{n}(r, s)$ is the $n$-dimensional annulus $\{r<|x|<s\}$ ).

Using the changes of coordinates just described the expressions of the two metrics $g_{1}$ and $g_{2}$ on the hollow domain become respectively

$$
\begin{aligned}
g_{1}(z, t, \theta)= & g_{i j}^{(1)} d z^{i} \otimes d z^{j}+u_{\varepsilon}^{(1)} \frac{4}{n-2}\left[\left(d t \otimes d t+g_{\lambda \mu}^{(1)} d \theta^{\lambda} \otimes d \theta^{\mu}\right)+g_{t \theta}^{(1)} d t \ltimes d \theta\right] \\
& +g_{i t}^{(1)} d z^{i} \otimes d t+g_{i \lambda}^{(1)} d z^{i} \otimes d \theta^{\lambda}
\end{aligned}
$$

and

$$
\begin{aligned}
g_{2}(z, t, \theta)= & g_{i j}^{(2)} d z^{i} \otimes d z^{j}+u_{\varepsilon}^{(2)} \frac{4}{n-2}\left[\left(d t \otimes d t+g_{\lambda \mu}^{(2)} d \theta^{\lambda} \otimes d \theta^{\mu}\right)+g_{t \theta}^{(2)} d t \ltimes d \theta\right] \\
& +g_{i t}^{(2)} d z^{i} \otimes d t+g_{i \lambda}^{(2)} d z^{i} \otimes d \theta^{\lambda},
\end{aligned}
$$

where by the compact notation $d t \ltimes d \theta$ we indicate the general component of the normal metric tensor (i.e., it involves $d t \otimes d t, d \theta^{\lambda} \otimes d \theta^{\mu}$ and $d t \otimes d \theta^{\lambda}$ components), whereas the coefficients $g_{t \theta}$ multiplied by $u_{\varepsilon}^{(i)} \frac{4}{n-2}, i=1,2$ represent the correction to the Euclidean metric $u_{\varepsilon}^{(i)} \frac{4}{n-2}\left[\left(d t \otimes d t+g_{\lambda \mu}^{(i)} d \theta^{\lambda} \otimes d \theta^{\mu}\right)\right], i=1,2$ in our coordinate system.

Remark that for $j=1,2$ we have

$$
\begin{array}{ll}
g_{\lambda \mu}^{(j)}=\mathcal{O}(1) & g_{t \theta}^{(j)}=\mathcal{O}\left(|x|^{2}\right) \\
g_{i t}^{(j)}=\mathcal{O}\left(|x|^{2}\right) & g_{i \lambda}^{(j)}=\mathcal{O}\left(|x|^{2}\right)
\end{array}
$$

and

$$
u_{\varepsilon}^{(1)}(t)=\varepsilon^{\frac{n-2}{2}} \mathrm{e}^{-\frac{n-2}{2} t} \quad \text { and } \quad u_{\varepsilon}^{(2)}(t)=\varepsilon^{\frac{n-2}{2}} \mathrm{e}^{\frac{n-2}{2} t} .
$$

We choose a cut-off function $\chi:(\log \varepsilon,-\log \varepsilon) \rightarrow[0,1]$ to be a non increasing smooth function which is identically equal to 1 in $(\log \varepsilon,-1]$ and 0 in $[1,-\log \varepsilon)$ and we choose another cut-off function $\eta:(\log \varepsilon,-\log \varepsilon) \rightarrow[0,1]$ to be a non increasing smooth function which is identically equal to 1 in $(\log \varepsilon,-\log \varepsilon-1]$ and which satisfies $\lim _{t \rightarrow-\log \varepsilon} \eta=0$. Using these two cut-off functions, we can define a new normal conformal factor $u_{\varepsilon}$ by

$$
u_{\varepsilon}(t):=\eta(t) u_{\varepsilon}^{(1)}(t)+\eta(-t) u_{\varepsilon}^{(2)}(t),
$$

and the metric $g_{\varepsilon}$ by

$$
\begin{aligned}
g_{\varepsilon}(z, t, \theta):= & \left(\chi g_{i j}^{(1)}+(1-\chi) g_{i j}^{(2)}\right) d z^{i} \otimes d z^{j} \\
& +u_{\varepsilon}^{\frac{4}{n-2}}\left[d t \otimes d t+\left(\chi g_{\lambda \mu}^{(1)}+(1-\chi) g_{\lambda \mu}^{(2)}\right) d \theta^{\lambda} \otimes d \theta^{\mu}\right. \\
& \left.+\left(\chi g_{t \theta}^{(1)}+(1-\chi) g_{t \theta}^{(2)}\right) d t \ltimes d \theta\right] \\
& +\left(\chi g_{i t}^{(1)}+(1-\chi) g_{i t}^{(2)}\right) d z^{i} \otimes d t \\
& +\left(\chi g_{i \lambda}^{(1)}+(1-\chi) g_{i \lambda}^{(2)}\right) d z^{i} \otimes d \theta^{\lambda} .
\end{aligned}
$$

Closer inspection of this expression shows that the only objects that are not a priori globally defined on the identification of the tubular neighborhoods (poly-neck) of $\iota_{1}(K)$ in $M_{1}$ and $\iota_{2}(K)$ in $M_{2}$ are the functions $\chi$ and $u_{\varepsilon}$ (since the cut-off $\eta$ is involved in the definition). However, observe that both cut-off functions can easily be expressed as functions of the 
Riemannian distance to $K$ in the respective manifolds. Hence they are globally defined and the metric $g_{\varepsilon}$-whose definition can be obviously completed by setting $g_{\varepsilon} \equiv g_{1}$ and $g_{\varepsilon} \equiv g_{2}$ out of the polyneck - is a Riemannian metric which is globally defined on the manifold $M_{\varepsilon}$.

We conclude this section with the definition of the proto-TT-tensor $\mu=\mu(\varepsilon)$, which is the symmetric $g_{\varepsilon}$-trace free tensor which will be corrected to a symmetric $g_{\varepsilon}$-TT-tensor by adding an $\varepsilon$-uniformly bounded term $\sigma_{\varepsilon}$. In order to do that we describe a warped cut off procedure on the side of the polyneck coming from $M_{1}$. The same manipulation on the other side provides us with the complete definition of $\mu$.

Let a $g_{1}$-trace-free symmetric tensor $\mu_{1}$ be given on $M_{1}$. In local coordinates this reads

$$
g_{i j}^{(1)} \mu_{1}^{i j}+2 g_{i \alpha}^{(1)} \mu_{1}^{i \alpha}+g_{\alpha \beta}^{(1)} \mu_{1}^{\alpha \beta}=0 .
$$

We are looking for a symmetric tensor $\mu$ which is trace free with respect to the metric $g_{\varepsilon}$. To do that we set

$$
\begin{aligned}
\mu^{i j} & =a(t) \cdot \mu_{1}^{i j} \\
\mu^{i \alpha} & =a(t) \cdot \mu_{1}^{i \alpha} \\
\mu^{\alpha \beta} & =b(t) \cdot \mu_{1}^{\alpha \beta},
\end{aligned}
$$

where $a$ and $b$ are smooth radial cut-off functions which are equal to one on $M_{1}$ and which vanish for $t>c \log \varepsilon+1$, where $0<c<1$ is a constant that will be determined in Sect. 4 . The definitions of $a$ and $b$ are made more precise below; however we remark that the warped cut-off still guarantees the symmetry of $\mu$. Taking into account (22) and the definition of the metric $g_{\varepsilon}$ on the region where $\mu$ is not identically zero, the condition $\operatorname{tr}_{g_{\varepsilon}} \mu=0$ is equivalent to

$$
\begin{aligned}
0 & =a g_{i j}^{(1)} \mu_{1}^{i j}+2 a g_{i \alpha}^{(1)} \mu_{1}^{i \alpha}+b \phi^{2} g_{\alpha \beta}^{(1)} \mu_{1}^{\alpha \beta} \\
& =\left[b \phi^{2}-a\right] \cdot g_{\alpha \beta}^{(1)} \mu_{1}^{\alpha \beta},
\end{aligned}
$$

where the normal conformal factor $\phi^{2}$ is by definition

$$
\phi^{2}:=\left[1+\eta(-t) \cdot\left(u_{\varepsilon}^{(2)} / u_{\varepsilon}^{(1)}\right)\right]^{\frac{4}{n-2}} .
$$

It is now straightforward to verify that one can always choose two smooth cut-off functions satisfying the conditions above and such that $a=\phi^{2} b$. In particular we will choose $b \equiv 1$, for $\log \varepsilon<t<c \log \varepsilon$. As a consequence, $a=b+\mathcal{O}\left(\mathrm{e}^{(n-2) t}\right)$ on this interval and $\mu$ converges to $\mu_{1}$ on the compact sets of $M_{1} \backslash K$ with respect to the $\mathcal{C}^{2}$-topology. Notice that since $\phi$ depends on $\varepsilon, a$ and $b$ do as well, but they admit an $\varepsilon$-uniform bound, as do their derivatives. Finally, let us observe that, for every $k \geq 0,\left|\nabla^{k} \operatorname{div}_{g_{\varepsilon}} \mu\right|_{g_{\varepsilon}}(t) \rightarrow\left|\nabla^{k} \operatorname{div}_{g_{1}} \mu_{1}\right|_{g_{1}}(\log \varepsilon)=0$, as $t$ tends to $\log \varepsilon$. Moreover $\left|\operatorname{div}_{g_{\varepsilon}} \mu\right|_{g_{\varepsilon}}$ and $\left|\nabla \operatorname{div}_{g_{\varepsilon}} \mu\right|_{g_{\varepsilon}}$ are $\mathcal{O}\left(\varepsilon^{n-2}\right)$ near the boundary of the polyneck and $\varepsilon$-uniformly bounded in the interior.

\section{The momentum constraint}

\subsection{The vector Laplacian $L_{g_{\varepsilon}}$}

As explained in the introduction, the next task is now to repair the momentum constraint. We start from $\mu$ defined in the previous section, which is symmetric and trace free, but in general does not satisfy the equation $\operatorname{div}_{g_{\varepsilon}} \mu=0$. We want to replace it by a symmetric divergence 
free tensor $\mu_{\varepsilon}$ whose $\varepsilon$-trace is still zero. The way to do that is to find a symmetric trace-free correction term $\sigma_{\varepsilon}$ whose norm $\left|\sigma_{\varepsilon}\right|_{g_{\varepsilon}}^{2}$ admits a suitable bound. This way we are allowed to choose $\mu_{\varepsilon}:=\mu+\sigma_{\varepsilon}$ to be a TT-tensor and to put the term $\left|\mu_{\varepsilon}\right|_{g_{\varepsilon}}^{2}$ in the nonlinear equation $\operatorname{Lic}_{g_{\varepsilon}} u=0$. Then the uniform bound enables us to get an appropriate estimate for the error term of the latter equation and then solve it by means of a perturbation argument.

As discussed above, a good way to proceed is to seek a correction term of the form $\sigma_{\varepsilon}=D_{g_{\varepsilon}} X$, where $D_{g_{\varepsilon}}$ is the conformal deformation operator with respect to the metric $g_{\varepsilon}$. This automatically guarantees that $\sigma_{\varepsilon}$ is symmetric and trace free, as it is easy to see from the local expression of this operator

$$
\left(D_{g_{\varepsilon}}\right)_{j k}=\frac{1}{2}\left[\left(\nabla_{j} X\right)_{k}+\left(\nabla_{k} X\right)_{j}\right]-\frac{1}{m}\left(\nabla_{l} X\right)^{l} \cdot g_{j k}^{(\varepsilon)},
$$

where $\nabla$ is the Levi-Civita connection of the metric $g_{\varepsilon}$, and the indices has been lowered by means of the metric $g_{\varepsilon}$, where needed.

The problem we are led to consider is then the vector equation

$$
L_{g_{\varepsilon}} X=\sharp \operatorname{div}_{g_{\varepsilon}} \mu,
$$

where the operator involved - the so called vector Laplacian—is defined as $L_{g_{\varepsilon}}:=\left(D_{g_{\varepsilon}}\right)^{*}$. $D_{g_{\varepsilon}}=-\sharp \operatorname{div}_{g_{\varepsilon}} \cdot D_{g_{\varepsilon}}$. As it is easy to verify, $L_{g_{\varepsilon}}$ is a linear elliptic second order partial differential operator with smooth coefficients and it is formally self-adjoint. We can think of the vector Laplacian as acting between the spaces of sections with Hölder regularity

$$
L_{g_{\varepsilon}}: \mathcal{C}^{2, \alpha}\left(M_{\varepsilon}, T M_{\varepsilon}\right) \longrightarrow \mathcal{C}^{0, \alpha}\left(M_{\varepsilon}, T M_{\varepsilon}\right)
$$

In Sect. 3.2, in order to produce an $\varepsilon$-uniform a priori bound for solutions to (25), we introduce a more sophisticated functional setting (i.e., weighted Hölder spaces of sections of fiber bundles); but now the following definitions are sufficient. For a general tensor field $T$ we define the $\mathcal{C}^{k}$ norm of $T$ with respect to a Riemmannian metric $g$ as

$$
{ }^{g}\|T\|_{\mathcal{C}^{k}}:=\sum_{j=0}^{k} \sup _{M}\left|\nabla^{j} T\right|_{g},
$$

where $\nabla$ is the Levi-Civita connection of $g$; we define the Hölder seminorm of the $k$-th derivative with exponent $\alpha \in(0,1)$ as

$$
g\left[\nabla^{k} T\right]_{\alpha}:=\sup _{p \neq q} \frac{\left|\nabla^{k} T(p)-\nabla^{k} T(q)\right|_{g}}{d_{g}(p, q)^{\alpha}},
$$

where the distance $d_{g}(p, q)$ is supposed to be smaller than the injectivity radius and with abuse of notation the term $\nabla^{k} T(q)$ is interpreted as its parallel transport from $q$ to $p$ along the unique geodesic joining $p$ and $q$ (in order to give sense to the subtraction which appears in the numerator above). The definition of the $\mathcal{C}^{k, \alpha}$-Hölder norm follows obviously.

The general theory of elliptic operators between vector bundles and in particular the Fredholm alternative guarantees the existence of a solution to the equation $L_{g_{\varepsilon}} X=W$, provided that the right hand term $W$ is orthogonal to $\operatorname{Ker} L_{g_{\varepsilon}}^{*}$. In our case we have to check the vanishing 
of the $L^{2}$-product

$$
\begin{aligned}
-\left\langle\sharp \operatorname{div}_{g_{\varepsilon}} \mu, Y\right\rangle & :=-\int_{M_{\varepsilon}} g_{\varepsilon}\left(\sharp \operatorname{div}_{g_{\varepsilon}} \mu, Y\right) \text { dvol }_{g_{\varepsilon}} \\
& =\int_{M_{\varepsilon}} g_{\varepsilon}\left(\mu, D_{g_{\varepsilon}} Y\right) d \operatorname{vol}_{g_{\varepsilon}},
\end{aligned}
$$

where $Y$ is an element of $\operatorname{Ker} L_{g_{\varepsilon}}^{*}=\operatorname{Ker} L_{g_{\varepsilon}}$, by self-adjointness. Since for each fixed $\varepsilon \in(0,1)$ the generalized connected sum $M_{\varepsilon}$ is a compact manifold, the integration by parts yields

$$
0=\left\langle L_{g_{\varepsilon}} Y, Y\right\rangle={ }^{\varepsilon}\left\|D_{g_{\varepsilon}} Y\right\|_{L^{2}}^{2},
$$

and the orthogonality is then proved. Hence, for each $\varepsilon \in(0,1)$ we can get a vector field $X_{\varepsilon}$ satisfying the equation (25).

\subsection{A priori uniform bound for solutions of $L_{g_{\varepsilon}} X=\sharp \operatorname{div}_{g_{\varepsilon}} \mu$}

This section is devoted to providing the existence of solutions $X_{\varepsilon}$ of the equations $L_{g_{\varepsilon}} X=\sharp \operatorname{div}_{g_{\varepsilon}} \mu$ with an a priori bound which is uniform in $\varepsilon \in(0,1)$. As noted earlier, a more sophisticated functional setting is needed. In particular the weighted Hölder spaces turn out to be the crucial tools needed to get the estimate we want. Using the definition of $t$ from Sect. 2, we define the distance function $\rho_{\varepsilon}$ to be $\rho_{\varepsilon}:=\varepsilon \cosh t$ for $(\log \varepsilon)+1<t<-(\log \varepsilon)-1$ (i.e., in the middle of the polyneck), to be $\rho_{\varepsilon} \equiv 1$ out of the radius one tubular neighborhoods of $K$ in $M_{1}$ and in $M_{2}$ and to be a monotone radial smooth interpolation in between these regions. Having introduced a radial distance function, we can define, for $k \in \mathbb{N}$ and $\alpha \in(0,1)$, the weighted $\mathcal{C}^{k}$-norms and the weighted Hölder $\alpha$-seminorms for a general tensor field $T$ on $M_{\varepsilon}$ with respect to the metric $g_{\varepsilon}$. The definition of the weighted $\mathcal{C}^{k, \alpha}$-Hölder norm follows at once. For a general weight $\gamma \in \mathbb{R}$ let us set

$$
\begin{aligned}
{ }^{\varepsilon}\|T\|_{\mathcal{C}_{\gamma}^{k}} & :=\sum_{j=0}^{k} \sup _{M_{\varepsilon}}\left\{\rho_{\varepsilon}^{-\gamma+j} \cdot\left|\nabla^{j} T\right|_{g_{\varepsilon}}\right\} \\
\varepsilon\left[\nabla^{k} T\right]_{\alpha, \gamma}: & =\sup _{p \neq q}\left\{\left|\rho_{\varepsilon}(p) \wedge \rho_{\varepsilon}(q)\right|^{-\gamma+k} \cdot \frac{\left|\nabla^{k} T(p)-\nabla^{k} T(q)\right|_{g_{\varepsilon}}}{d_{g_{\varepsilon}}(p, q)^{\alpha}}\right\},
\end{aligned}
$$

where $\nabla$ indicates the Levi-Civita connection of $g_{\varepsilon},\left|\rho_{\varepsilon}(p) \wedge \rho_{\varepsilon}(q)\right|$ is the minimum between $\rho_{\varepsilon}(p)$ and $\rho_{\varepsilon}(q)$, and the conventions used in (28) are still valid for (30). In the following we indicate by $\rho_{\varepsilon}^{\gamma} \cdot \mathcal{C}^{k, \alpha}\left(M_{\varepsilon}, T M_{\varepsilon}\right)$ the space of tensor fields $X$ such that the norm ${ }^{\varepsilon}\|X\|_{\mathcal{C}_{\gamma}^{k, \alpha}}:={ }^{\varepsilon}\|X\|_{\mathcal{C}_{\gamma}^{k}}+{ }^{\varepsilon}\left[\nabla^{k} X\right]_{\alpha, \gamma}$ is well defined and finite. In this context it is convenient to think of $L_{g_{\varepsilon}}$ as acting between the spaces

$$
L_{g_{\varepsilon}}: \rho_{\varepsilon}^{\delta} \cdot \mathcal{C}^{2, \alpha}\left(M_{\varepsilon}, T M_{\varepsilon}\right) \longrightarrow \rho_{\varepsilon}^{\delta-2} \cdot \mathcal{C}^{0, \alpha}\left(M_{\varepsilon}, T M_{\varepsilon}\right),
$$

for a suitable weight $\delta \in \mathbb{R}$. Notice that, for fixed $\varepsilon$, the functional setting of (31) is strictly equivalent to the one of (26). In particular, the existence result of the previous section still holds. The reason for introducing weighted spaces is that uniform estimates are not available in the old context, since the geometry of our construction becomes singular when the parameter $\varepsilon$ tends to zero. Having introduced these new analytical devices, we can now state the following: 
Proposition 2 Let $X \in \rho_{\varepsilon}^{\delta} \cdot \mathcal{C}^{2, \alpha}\left(M_{\varepsilon}, T M_{\varepsilon}\right)$ and $W \in \rho_{\varepsilon}^{\delta-2} \cdot \mathcal{C}^{0, \alpha}\left(M_{\varepsilon}, T M_{\varepsilon}\right)$ be vector fields satisfying the equation $L_{g_{\varepsilon}} X=W$. Moreover suppose that $W$ is of the form $W=\sharp \operatorname{div}_{g_{\varepsilon}} \mu$, for some symmetric 2-tensor $\mu$. Then, if the weight $\delta$ is chosen to be in $(2-n,(2-n) / 2)$, there exists a constant $C>0$ independent of $\varepsilon \in(0,1)$ such that

$$
{ }^{\varepsilon}\|X\|_{\mathcal{C}_{\delta}^{1}} \leq C \cdot{ }^{\varepsilon}\|W\|_{\mathcal{C}_{\delta-2}^{0, \alpha}}
$$

(Remember that $n$ is the codimension of $K$ in $M_{i}, i=1,2$ and it is supposed to be greater than 3).

The proof is by contradiction. If such a constant $C$ does not exist, we can find out for every $j \in \mathbb{N}$ a triple $\left(\varepsilon_{j}, X_{j}, W_{j}\right)$ such that

1. $\varepsilon_{j} \rightarrow 0$ as $j \rightarrow+\infty$

2. $L_{\varepsilon_{j}} X_{j}=W_{j} \quad$ for every $j \in \mathbb{N}$

3. ${ }^{\varepsilon}{ }_{j}\left\|X_{j}\right\|_{\mathcal{C}_{\delta}^{1}}=1$ for every $j \in \mathbb{N}$

4. ${ }^{\varepsilon} j\left\|W_{j}\right\|_{\mathcal{C}_{\delta-2}^{0, \alpha}} \rightarrow 0 \quad$ as $j \rightarrow+\infty$

For technical reasons we prefer to replace condition 3. with the following

3.bis $\sup _{M_{j}}\left\{\rho_{j}^{-\delta} \cdot\left|X_{j}\right|_{\varepsilon_{j}}+\rho_{j}^{-\delta+1} \cdot\left|\nabla X_{j}\right|_{\varepsilon_{j}}\right\}=1, \quad$ for every $j \in \mathbb{N}$.

Notice that this can be done because condition 3.bis is nothing but an equivalent way to define the norm ${ }^{\varepsilon_{j}}\left\|X_{j}\right\|_{\mathcal{C}_{\delta}^{1}}$. Since each $M_{j}$ is compact we can look now at the points $p_{j}$ 's where this maximum is achieved, so that

$$
\left\{\rho_{j}^{-\delta} \cdot\left|X_{j}\right|_{\varepsilon_{j}}+\rho_{j}^{-\delta+1} \cdot|\nabla X|_{\varepsilon_{j}}\right\}\left(p_{j}\right)=1
$$

To carry out the proof we are going to take the limit of the expression $\mathbf{2}$. It is then clear that the issue reduces to investigating whether the homogeneous limit problem admits nontrivial solutions with prescribed decay, where the non triviality strictly depends on the behavior at the limit of the $p_{j}$ 's and the decay is prescribed by the weight. This leads us to distinguish three different limit situations, depending on how the geometric structure degenerates near the $p_{j}$ 's, as $j \rightarrow+\infty$.

Case 1. The $p_{j}$ 's converge, up to a subsequence, to a point $p_{\infty}$ which lies in $M_{1} \backslash K$ (or analogously in $M_{2} \backslash K$ ), as $j \rightarrow+\infty$. In this case, as we discuss below, we are induced to look for nontrivial solutions of the homogeneous problem

$$
\left\{\begin{array}{l}
L_{g_{1}} X=0 \quad \text { on } M_{1} \backslash K \\
|X|_{g_{1}} \leq A \cdot r^{\delta} \\
|\nabla X|_{g_{1}} \leq A \cdot r^{\delta-1}
\end{array}\right.
$$

where $r:=d_{g_{1}}(\cdot, K)$, and $A>0$ is a positive constant.

Case 2. The $p_{j}$ 's converge, up to a subsequence, to a point $p_{\infty}$ which lies in $K$ with the same speed as the radius of the excised tubular neighborhood: $d_{g_{1}}\left(p_{j}, K\right)=\mathcal{O}\left(\varepsilon_{j}\right)$, as $j \rightarrow+\infty$ (notice that, up to a subsequence, we can suppose without loss of generality that all the $p_{j}$ 's lie in the side coming from $M_{1}$ ). In this case, by means of a blow-up method, we are induced to look for nontrivial solutions of the 
homogeneous problem

$$
\left\{\begin{array}{l}
L_{\mathbb{R}^{k} \times \mathbb{S}^{n}} X=0 \\
|X|_{\mathbb{R}^{k} \times \mathbb{S}^{n}} \leq B \cdot(\cosh t)^{\delta} \\
|\nabla X|_{\mathbb{R}^{k} \times \mathbb{S}^{n}} \leq B \cdot(\cosh t)^{\delta-1}
\end{array} \quad \text { on } \mathbb{R}_{z}^{k} \times \mathbb{S}_{t, \theta}^{n}\right.
$$

where $B>0$ is a positive constant and $\mathbb{S}^{n}$ denotes the $n$-dimensional Schwarzschild space. Moreover, by the expressions $L_{\mathbb{R}^{k} \times \mathbb{S}^{n}}$ and $|\cdot|_{\mathbb{R}^{k} \times \mathbb{S}^{n}}$, we indicate respectively the vector Laplacian and the norm of the product metric $g_{\mathbb{R}^{k}}+g_{\mathbb{S}^{n}}$.

Case 3. The $p_{j}$ 's converge, up to a subsequence, to a point $p_{\infty}$ which lies in $K$ with a lower speed than the radius of the excised tubular neighborhood: $d_{g_{1}}\left(p_{j}, K\right) / \varepsilon_{j} \rightarrow+\infty$, as $j \rightarrow+\infty$ (notice that, up to a subsequence, we can suppose without loss of generality that all the $p_{j}$ 's lie in the side coming from $M_{1}$ ). In this case, refining the blow-up method of the previous case, we are induced to look for nontrivial solutions of the homogeneous problem

$$
\left\{\begin{array}{l}
L_{\mathbb{R}^{k} \times \mathbb{R}^{n}} X=0 \quad \text { on } \mathbb{R}_{z}^{k} \times\left(\mathbb{R}_{x}^{n} \backslash\{0\}\right) \\
|X|_{\mathbb{R}^{k} \times \mathbb{R}^{n}} \leq C \cdot|x|^{\delta} \\
|\nabla X|_{\mathbb{R}^{k} \times \mathbb{R}^{n}} \leq C \cdot|x|^{\delta-1}
\end{array}\right.
$$

where $C>0$ is a positive constant.

The choice of the weight $\delta$ in the right interval $(2-n,(2-n) / 2)$ leads us to a contradiction in all of the three cases. In other words the homogeneous problems with prescribed decay (34)-(36) only admit trivial solutions. In the following we analyze one by one the three cases presented above.

\subsubsection{Case 1: the equation $L_{g_{1}} X=0$ and the non-degeneracy condition}

In this first case we assume that (up to a subsequence) the $p_{j}$ 's tend to a point $p_{\infty} \in M_{1} \backslash K$. As it is easy to check from the expression (21), we have that the metrics $g_{\varepsilon_{j}}$ 's converge to the metric $g_{1}$ with respect to to the $\mathcal{C}^{2}$-topology on the compact sets of $M_{1} \backslash K$. Hence on a fixed compact set $Q \subset M_{1} \backslash K$ the weighted $g_{\varepsilon_{j}}$ norms are all equivalent to the standard $g_{1}$ norm. More precisely, there exist two positive constants $A(Q), B(Q)>0$ such that

$$
A(Q) \cdot{ }^{\varepsilon_{j}}\|X\|_{\mathcal{C}_{\delta}^{1}(Q)} \leq{ }^{g_{1}}\|X\|_{\mathcal{C}^{1}(Q)} \leq B(Q) \cdot{ }^{\varepsilon_{j}}\|X\|_{\mathcal{C}_{\delta}^{1}(Q)}
$$

In particular, if $p_{\infty} \in Q$, we have that for every sufficiently large $j \in \mathbb{N}$

$$
A(Q) \leq{ }^{g_{1}}\left\|X_{j}\right\|_{\mathcal{C}^{1}(Q)} \leq B(Q) .
$$

Our task is now to show that the vector fields $X_{j}$ converge to a nontrivial vector field $X$ with respect to the $\mathcal{C}^{1}(Q)$ topology. Thanks to the Ascoli-Arzelá Theorem and to the estimate (38), our goal is achieved if we can produce a $j$-uniform bound for ${ }^{g_{1}}\left\|X_{j}\right\|_{\mathcal{C}^{1, \alpha}(Q)}$. In order to do that we invoke the following result from [6], which is a Schauder interior estimate for second order linear elliptic systems in divergence form. 
Proposition 3 Let $X \in W_{\text {loc }}^{1,2}(\Omega)$ be a solution to

$$
\nabla_{\sigma}\left(A_{i j}^{\sigma \tau} \nabla_{\tau} X^{j}\right)=-\nabla_{\sigma} F_{i}^{\sigma}
$$

with $A_{i j}^{\sigma \tau} \in \mathcal{C}^{0, \alpha}(\Omega)$, for $1 \leq i, j \leq m$ and $1 \leq \sigma, \tau \leq 2$, satisfying the Legendre-Hadamard condition

$$
A_{i j}^{\sigma \tau} \xi_{\sigma} \xi_{\tau} \eta^{i} \eta^{j} \geq \lambda|\xi|^{2}|\eta|^{2} \quad, \forall \xi \in \mathbb{R}^{2}, \forall \eta \in \mathbb{R}^{n}
$$

If $F_{\sigma}^{i} \in \mathcal{C}^{0, \alpha}(\Omega)$, then we have $\nabla X \in \mathcal{C}^{0, \alpha}(\Omega), \alpha \in(0,1)$. Moreover, for every compact set $K \subset \Omega$

$$
\|\nabla X\|_{\mathcal{C}^{0, \alpha}(K)} \leq C \cdot\left\{\|\nabla X\|_{L^{2}(\Omega)}+\|F\|_{\mathcal{C}^{0, \alpha}(\Omega)}\right\},
$$

with $C$ depending on $K$, the ellipticity constant $\lambda$ and the Hölder norm of the coefficients $A_{i j}^{\sigma \tau}$.

Since $Q$ is compact we can cover it with finitely many small balls, and then we can cover each of these balls with another ball with a little larger radius. Using Proposition 3, we can easily get the desired $j$-uniform $\mathcal{C}^{1, \alpha}$ bound on each small ball. In fact, since the metrics $g_{\varepsilon_{j}}$ 's converge to $g_{1}$, the coefficients of the operators $L_{g_{\varepsilon_{j}}}$ too will converge to the coefficients of $L_{g_{1}}$. In particular the ellipticity constant can be chosen to be the same and consequently also the constant in the estimate (39) can be chosen to be the same. Up to taking the maximum of the finitely many constants obtained as a new constant, we have that there exists a real number $C>0$ depending only on the compact set $Q$ and on the ellipticity constant of the operator $L_{g_{1}}$ on $Q$, such that

$$
{ }^{g_{1}}\left\|X_{j}\right\|_{\mathcal{C}^{1, \alpha}(Q)} \leq C \cdot\left\{{ }^{g_{1}}\left\|X_{j}\right\|_{\mathcal{C}^{0}\left(Q^{\prime}\right)}+{ }^{g_{1}}\left\|W_{j}\right\|_{\mathcal{C}^{0, \alpha}\left(Q^{\prime}\right)}\right\}
$$

where $Q^{\prime}$ is another compact set of $M_{1} \backslash K$ including $Q$ and the larger small balls. Hence, taking into account the hypothesis of the argument by contradiction and the inequality (38), there must exists a constant $C^{\prime}>0$ depending on $C$ and $Q$ such that

$$
{ }^{g_{1}}\left\|X_{j}\right\|_{\mathcal{C}^{1, \alpha}(Q)} \leq C^{\prime} .
$$

Hence we can conclude that there exists a vector field $X$ defined on $M_{1} \backslash K$ such that, up to a subsequence, $X_{j} \rightarrow X$ with respect to the $\mathcal{C}^{1}$-topology on the compact sets of $M_{1} \backslash K$. Therefore $X$ must satisfy the homogeneous problem (34). Moreover, the inequality (38) guarantees that $X$ is non identically zero.

As it is easy to verify, the condition $2-n<\delta$ guarantees that $X$ verifies the equation $L_{g_{1}} X=0$ on the whole $M_{1}$ in the sense of distributions, and by elliptic regularity we deduce that $X$ is smooth. Since $M_{1}$ is compact, we can integrate by parts obtaining

$$
0=\left\langle L_{g_{1}} X, X\right\rangle={ }^{g_{1}}\left\|D_{g_{1}} X\right\|_{L^{2}}^{2} .
$$

Hence $X$ is a nontrivial conformal Killing vector field on $M_{1}$, which is excluded by the non-degeneracy condition.

\subsubsection{Case 2: the equation $L_{\mathbb{R}^{k} \times \mathbb{S}^{n}} X=0$ and the blow-up method}

In this case we suppose that up to a subsequence the $p_{j}$ 's tend to a point $p_{\infty} \in K$ lying on the side of $M_{1}$ with the higher velocity allowed (i.e. $\left.d_{g_{1}}\left(p_{j}, p_{\infty}\right)=\mathcal{O}\left(\varepsilon_{j}\right)\right)$. Since as $\varepsilon \rightarrow 0$ the geometry of our construction becomes singular we are induced to perform a 
blow-up around the point $p_{\infty}$ in order to investigate the analytic behavior of the objects we are considering. Let us fix then a local system of Fermi coordinates centered at $p_{\infty}$, so that $z\left(p_{\infty}\right)=0=x\left(p_{\infty}\right)$ and let us focus on the region $B^{k}(1) \times A^{n}(\varepsilon, 1)$, where $B^{k}(1)$ and $A^{n}(\varepsilon, 1)$ are respectively the $k$-dimensional unit ball and the $n$-dimensional annulus centered at $p_{\infty}$. We introduce now a family of diffeomorphisms $\left(\phi_{\varepsilon}\right)_{\varepsilon \in(0,1)}$, defined as

$$
\begin{aligned}
\phi_{\varepsilon}: B^{k}(1 / \varepsilon) \times A^{n}(1,1 / \varepsilon) & \longrightarrow B^{k}(1) \times A^{n}(\varepsilon, 1) \\
(z, x) & \longmapsto(\varepsilon z, \varepsilon x) .
\end{aligned}
$$

Using the $\phi_{\varepsilon}$ 's, we define on the new domains the blow-up metrics $\stackrel{\circ}{g_{\varepsilon}}$ by setting

$$
\stackrel{\circ}{g_{\varepsilon}}:=\frac{1}{\varepsilon^{2}} \cdot\left[\phi_{\varepsilon}^{*} g_{\varepsilon}\right]
$$

In the limit for $\varepsilon \rightarrow 0$ the domain of definition of the $\phi_{\varepsilon}$ 's becomes $\mathbb{R}^{k} \times\left(\mathbb{R}^{n} \backslash B^{n}(1)\right)$ and the blow-up metrics $\stackrel{\circ}{g}_{\varepsilon}$ tend to $g_{\mathbb{R}^{k}}+g_{\mathbb{S}^{n}}$ on the compact sets of $\mathbb{R}^{k} \times\left(\mathbb{R}^{n} \backslash B^{n}(1)\right)$ in the $\mathcal{C}^{2}$ topology. In fact the local expression for $\stackrel{\circ}{g}_{\varepsilon}$ reads

$$
\begin{aligned}
\stackrel{\circ}{g_{\varepsilon}}(z, x)= & \frac{1}{\varepsilon^{2}} \cdot\left\{g_{i j}^{K}(\varepsilon z)+\mathcal{O}(\varepsilon|x|)\right\} \cdot d\left(\varepsilon z^{i}\right) \otimes d\left(\varepsilon z^{j}\right) \\
& +\frac{1}{\varepsilon^{2}} \cdot \mathcal{O}(\varepsilon|x|) \cdot d\left(\varepsilon z^{i}\right) \otimes d\left(\varepsilon x^{\alpha}\right) \\
& +\frac{1}{\varepsilon^{2}} \cdot\left(|x|^{\frac{n-2}{2}}+|x|^{-\frac{n-2}{2}}\right)^{\frac{4}{n-2}} \cdot\left\{|x|^{-2} \delta_{\alpha \beta}+\mathcal{O}\left(\varepsilon^{2}\right)\right\} \cdot d\left(\varepsilon x^{\alpha}\right) \otimes d\left(\varepsilon x^{\beta}\right) \\
= & \left\{\delta_{i j}+\mathcal{O}(\varepsilon|x|)+\mathcal{O}\left(\varepsilon^{2}|z|^{2}\right)\right\} \cdot d z^{i} \otimes d z^{j} \\
& +\mathcal{O}(\varepsilon|x|) \cdot d z^{i} \otimes d x^{\alpha} \\
& +\left\{g_{\alpha \beta}^{\mathbb{S}^{n}}(x)+\mathcal{O}\left(\varepsilon^{2}\right)\right\} \cdot d x^{\alpha} \otimes d x^{\beta} .
\end{aligned}
$$

(Notice that the blow-up construction described here obviously applies to both the sides of the polyneck in order to get the whole Schwarzschild space as limit manifold and to the operator $L_{\mathbb{R}^{k} \times \mathbb{S}^{n}}$ as limit operator. The description of the blow-up procedure in terms of $(z, x)$ coordinates makes clearer the analogies and the differences between this blow-up and the one we use in treating the third case. Nevertheless it is possible to give the description of the same procedure in terms of $(z, t, \theta)$ coordinates. In this case it is sufficient to remember that for the metric $g_{\varepsilon}$ we have at hand the expression

$$
\begin{aligned}
g_{\varepsilon}(z, t, \theta):= & \left\{\delta_{i j}+\mathcal{O}(\varepsilon \cosh t) \mathcal{O}\left(|z|^{2}\right)\right\} \cdot d z^{i} \otimes d z^{j} \\
& +\left[\varepsilon^{\frac{n-2}{2}} \cosh \left(\frac{n-2}{2} t\right)\right]^{\frac{4}{n-2}}\left\{d t^{2}+d \theta^{2}+\mathcal{O}\left(\varepsilon^{2} \cosh ^{2} t\right) d t \ltimes d \theta\right\} \\
& +\mathcal{O}\left(\varepsilon^{2} \cosh ^{2} t\right) \cdot d z^{i} \otimes d t \\
& +\mathcal{O}\left(\varepsilon^{2} \cosh ^{2} t\right) \cdot d z^{i} \otimes d \theta^{\lambda},
\end{aligned}
$$

where $d \theta^{2}$ is the round metric on $S^{n-1}$. Then using the blow-up diffeomorphisms

$$
\begin{aligned}
\psi_{\varepsilon}: B^{k}(1 / \varepsilon) \times(\log \varepsilon,-\log \varepsilon) \times S^{n-1} & \longrightarrow B^{k}(1) \times(\log \varepsilon,-\log \varepsilon) \times S^{n-1} \\
(z, t, \theta) & \longmapsto(\varepsilon z, t, \theta)
\end{aligned}
$$


and defining the blow-up metrics as

$$
\stackrel{\circ}{g}_{\varepsilon}:=\frac{1}{\varepsilon^{2}} \cdot\left[\psi_{\varepsilon}^{*} g_{\varepsilon}\right]
$$

one can easily obtain the same results). Hence the coefficients of the operators $L_{g_{\varepsilon}}$ tend to the coefficients of the operator $L_{\mathbb{R}^{k} \times \mathbb{S}^{n}}$. Moreover, if we consider for every $j \in \mathbb{N}$ the vector fields

$$
\begin{aligned}
\stackrel{\circ}{X}_{j} & :=\varepsilon_{j}^{-\delta+1} \phi_{\varepsilon_{j}}^{*} \cdot X_{j} \\
\stackrel{\circ}{W}_{j} & :=\varepsilon_{j}^{-\delta+1} \phi_{\varepsilon_{j}}^{*} \cdot W_{j},
\end{aligned}
$$

we have that the triples $\left(\varepsilon_{j}, \stackrel{\circ}{X}_{j}, \stackrel{\circ}{W}_{j}\right)$ verify the properties $\mathbf{1}$ - - 4. with respect to the blow-up metrics $g_{\varepsilon_{j}}^{\circ}$ and the distance function(s)

$$
\rho_{\varepsilon_{j}}^{\circ}(t):=\cosh t .
$$

Using the same argument as in the previous section it is easy to show that the vector fields $\stackrel{\circ}{X}_{j}$ converge on the compact sets to a vector field $X$ with respect to the $\mathcal{C}^{1}$-topology of the metric $g_{\mathbb{R}^{K}}+g_{\mathbb{S}^{n}}$ and the sequence of problems

$$
\stackrel{\circ}{L_{j}} \stackrel{\circ}{X}_{j}=\stackrel{\circ}{W}_{j}
$$

converges to the homogeneous problem (35).

Moreover, since $d_{g_{1}}\left(p_{j}, K\right)=\mathcal{O}\left(\varepsilon_{j}\right)$, we have that the sequence of points $q_{j}:=\phi_{\varepsilon_{j}}^{-1}\left(p_{j}\right)$ lies in a compact region of $\mathbb{R}^{k} \times\left(\mathbb{R}^{n} \backslash B^{n}(1)\right)$ and converges, up to a subsequence, to a point $q_{\infty}$, so that the solution of the limit problem $X$ must be non identically zero.

Our task is now to show that the homogeneous problem (35) for the operator $L_{\mathbb{R}^{k} \times \mathbb{S}^{n}}$ with prescribed decay does not admit nontrivial solutions. In order to do that we write down the explicit expression of our equation, using the fact that we are dealing with the product metric $g_{\mathbb{R}^{k}}+g_{\mathbb{S}^{n}}$. If we set $X(z, x)=U(z, x)+V(z, x)$ with $U(z, x) \in \mathbb{R}^{k}$ and $V(z, x) \in T_{x} \mathbb{S}^{n}$, the vector Laplacian decomposes as follows

$$
\begin{aligned}
{\left[L_{\mathbb{R}^{k} \times \mathbb{S}^{n}} X\right]^{\mathbb{R}^{k}=} } & L_{\mathbb{R}^{k}} U-\left(\frac{m-k}{m \cdot k}\right) \cdot \operatorname{grad}_{\mathbb{R}^{k}} \circ \operatorname{div}_{\mathbb{R}^{k}} U \\
& -\frac{1}{2} \cdot \Delta_{\mathbb{S}^{n}} U-\left(\frac{m-2}{m \cdot 2}\right) \cdot \operatorname{grad}_{\mathbb{R}^{k}} \circ \operatorname{div}_{\mathbb{S}^{n}} V \\
{\left[L_{\mathbb{R}^{k} \times \mathbb{S}^{n}} X\right]^{T \mathbb{S}^{n}=} } & L_{\mathbb{S}^{n}} V-\left(\frac{m-n}{m \cdot n}\right) \cdot \operatorname{grad}_{\mathbb{S}^{n}} \circ \operatorname{div}_{\mathbb{S}^{n}} V \\
& -\frac{1}{2} \cdot \Delta_{\mathbb{R}^{k}} V-\left(\frac{m-2}{m \cdot 2}\right) \cdot \operatorname{grad}_{\mathbb{S}^{n}} \circ \operatorname{div}_{\mathbb{R}^{k}} U,
\end{aligned}
$$

where in general we indicate by $\Delta_{g} W$ the (negative definite) Laplace-Beltrami operator of a Riemannian metric $g$ applied to the components of the vector field $W$.

The idea we mean to use to carry out the analysis of this operator is to perform a Fourier transform along the $\mathbb{R}^{k}$ components. Then we will use the decay conditions along the $\mathbb{S}^{n}$ factor to deduce the injectivity of our operator via an integration by parts.

In order to fix a suitable functional setting, we define for $\beta \in \mathbb{R}$ and $l \in \mathbb{N}$ the weighted Sobolev space $H_{\beta}^{l}\left(\mathbb{S}^{n}, \mathbb{R}^{k} \otimes T \mathbb{S}^{n}\right)$ as the space of the sections $Y$ of the bundle $\mathbb{R}^{k} \otimes T \mathbb{S}^{n} \rightarrow \mathbb{S}^{n}$ 
such that the following expression

$$
\|Y\|_{H_{\beta}^{l}}^{2}:=\sum_{j=0}^{l} \int_{\mathbb{S}^{n}}\left|\nabla^{j} Y\right|_{\mathbb{R}^{k} \times \mathbb{S}^{n}}^{2} \cdot(\cosh t)^{-2 \beta+2 j} \text { dvol }_{\mathbb{S}^{n}}
$$

is well defined and finite.

Notice that if $X$ verifies the decay conditions prescribed in (35), then, for every $z \in \mathbb{R}^{k}$ and every weight $\gamma>\delta, X(z) \in H_{\gamma+n / 2}^{1}\left(\mathbb{S}^{n}, \mathbb{R}^{k} \otimes T \mathbb{S}^{n}\right)$. Moreover the $H_{\gamma+n / 2}^{1}$-norms of $X(z)$ are uniformly bounded with respect to $z \in \mathbb{R}^{k}$, i.e.,

$$
\sup _{z \in \mathbb{R}^{k}}\left|\|X(z)\|_{H_{\gamma+n / 2}^{1}}\right|<+\infty .
$$

Rephrasing this, we can say that

$$
X \in L^{\infty}\left(\mathbb{R}^{k}, H_{\gamma+n / 2}^{1}\left(\mathbb{S}^{n}, \mathbb{R}^{k} \otimes T \mathbb{S}^{n}\right)\right),
$$

for every $\gamma>\delta$.

To proceed, we define the Schwartz space $\mathcal{S}\left(\mathbb{R}^{k}, H_{\beta}^{l}\left(\mathbb{S}^{n}, \mathbb{R}^{k} \otimes T \mathbb{S}^{n}\right)\right)$ as the space of the vector fields $W$ such that the seminorms

$$
|W|_{r, s}^{l, \beta}:=\sup _{z \in \mathbb{R}^{k}}\left|z^{r} \cdot \partial_{z}^{s}\|W(z)\|_{H_{\beta}^{l}}\right|
$$

are well defined and finite for every couple of multi-indices $r, s \in \mathbb{N}^{k}$.

Having at hand these definitions, we observe that our solution $X$ defines, for every $\gamma>\delta$, a continuous linear functional on the space $\mathcal{S}\left(\mathbb{R}^{k}, H_{-\gamma-n / 2}^{1}\left(\mathbb{S}^{n}, \mathbb{R}^{k} \otimes T \mathbb{S}^{n}\right)\right)$ via the following natural assignment

$$
Y \mapsto\langle X, Y\rangle:=\int_{\mathbb{R}^{k}} \int_{\mathbb{S}^{n}} g_{\mathbb{R}^{k} \times \mathbb{S}^{n}}(X, Y) \operatorname{dvol}_{\mathbb{S}^{n}} d v o l_{\mathbb{R}^{k}},
$$

In other words, we can think of $X$ as a tempered distribution, namely

$$
X \in \mathcal{S}^{\prime}\left(\mathbb{R}^{k}, H_{\gamma+n / 2}^{1}\left(\mathbb{S}^{n}, \mathbb{R}^{k} \otimes T \mathbb{S}^{n}\right)\right),
$$

and $\langle\cdot, \cdot \cdot\rangle$ is nothing but the natural pairing on $\mathcal{S}^{\prime}\left(\mathbb{R}^{k}, H_{\gamma+n / 2}^{1}\right) \times \mathcal{S}\left(\mathbb{R}^{k}, H_{-\gamma-n / 2}^{1}\right)$, where here and in the following we indicate the space $H_{\beta}^{l}\left(\mathbb{S}^{n}, \mathbb{R}^{k} \otimes T \mathbb{S}^{n}\right)$ simply by $H_{\beta}^{l}$, for $l \in \mathbb{N}$ and $\beta \in \mathbb{R}$.

Now it is quite natural to define the formal adjoint $L_{\mathbb{R}^{k} \times \mathbb{S}^{n}}^{*}$ of our operator as

$$
L_{\mathbb{R}^{k} \times \mathbb{S}^{n}}^{*}: \mathcal{S}\left(\mathbb{R}^{k}, H_{-\gamma-n / 2+2}^{1}\right) \rightarrow \mathcal{S}\left(\mathbb{R}^{k}, H_{-\gamma-n / 2}^{1}\right),
$$

where $L_{\mathbb{R}^{k} \times \mathbb{S}^{n}}^{*} W=Y$ if and only if

$$
\int_{\mathbb{R}^{k}} \int_{\mathbb{S}^{n}}\left(D_{\mathbb{R}^{k} \times \mathbb{S}^{n}} W, D_{\mathbb{R}^{k} \times \mathbb{S}^{n}} Z\right) \operatorname{dvol}_{\mathbb{S}^{n}} d v o l_{\mathbb{R}^{k}}=\int_{\mathbb{R}^{k}} \int_{\mathbb{S}^{n}}(Y, Z) \operatorname{dvol}_{\mathbb{S}^{n}} \operatorname{dvol}_{\mathbb{R}^{k}},
$$

for every $Z \in \mathcal{S}\left(\mathbb{R}^{k}, H_{\gamma+n / 2}^{1}\right)$.

With this interpretation, the fact that $L_{\mathbb{R}^{k} \times \mathbb{S}^{n}} X=0$ amounts to say that

$$
\left\langle X, L_{\mathbb{R}^{k} \times \mathbb{S}^{n}}^{*} W\right\rangle=0,
$$

for every $W \in \mathcal{S}\left(\mathbb{R}^{k}, H_{-\gamma-n / 2+2}^{1}\right)$. 
Hence, if we prove that $L_{\mathbb{R}^{k} \times \mathbb{S}^{n}}^{*}$ is surjective, then $X=0$ and we get the contradiction. Therefore our task is to show that the equation

$$
L_{\mathbb{R}^{k} \times \mathbb{S}^{n}}^{*} W=Y
$$

admits a solution $W \in \mathcal{S}\left(\mathbb{R}^{k}, H_{-\gamma-n / 2+2}^{1}\right)$ for every $Y \in \mathcal{S}\left(\mathbb{R}^{k}, H_{-\gamma-n / 2}^{1}\right)$.

To prove that, we perform a Fourier transform along the $\mathbb{R}^{k}$ components. More precisely, using the orthogonal split $Y(z, x)=\Phi(z, x)+\Psi(z, x)$, with $\Phi(z, x)=\Phi^{j}(z, x) \cdot \mathrm{e}_{j} \in \mathbb{R}^{k}$ and $\Psi(z, x)=\Psi^{\alpha}(z, x) \cdot \partial_{x^{\alpha}} \in T_{x} \mathbb{S}^{n}$, then the partial Fourier transform of $Y$ is given by

$$
\widehat{Y}(\zeta, x)=\widehat{\Phi}^{j}(\zeta, x) \cdot \mathrm{e}_{j}+\widehat{\Psi}^{\alpha}(\zeta, x) \cdot \partial_{x^{\alpha}},
$$

where

$$
\begin{aligned}
& \widehat{\Phi}^{j}(\zeta, x):=(2 \pi)^{-k / 2} \int_{\mathbb{R}^{k}} \Phi^{j}(z, x) \cdot \mathrm{e}^{-i\langle\zeta, z\rangle} d z, \\
& \widehat{\Phi}^{\alpha}(\zeta, x):=(2 \pi)^{-k / 2} \int_{\mathbb{R}^{k}} \Psi^{\alpha}(z, x) \cdot \mathrm{e}^{-i\langle\zeta, z\rangle} d z .
\end{aligned}
$$

By means of this procedure, we can reduce the study of (53) to prove that the operators

$$
\widehat{L}_{\mathbb{R}^{k} \times \mathbb{S}^{n}}^{*}(\zeta): H_{-\gamma-n / 2+2}^{1} \rightarrow H_{-\gamma-n / 2}^{1}
$$

are surjective for every $\zeta \in \mathbb{R}^{k}$. Notice that in this expression we use the notation $H_{\beta}^{l}$ to indicate the space $H_{\beta}^{l}\left(\mathbb{S}^{n}, \mathbb{C}^{k} \otimes T^{\mathbb{C}^{n}}\right)$, for $l \in \mathbb{N}$ and $\beta \in \mathbb{R}$, since the partial Fourier transform leads us to consider the complexified tangent bundle $\mathbb{C}^{k} \otimes T^{\mathbb{C}} \mathbb{S}^{n} \rightarrow \mathbb{S}^{n}$ instead of $\mathbb{R}^{k} \otimes T \mathbb{S}^{n} \rightarrow \mathbb{S}^{n}$. In particular, if we write $\widehat{W}(\zeta, \cdot) \in H_{-\gamma-n / 2+2}^{1}$ as $\widehat{W}(\zeta, \cdot)=U+V$, with $U \in H_{-\gamma-n / 2+2}^{1}\left(\mathbb{S}^{n}, \mathbb{C}^{k}\right)$ and $V \in H_{-\gamma-n / 2+2}^{1}\left(\mathbb{S}^{n}, T^{\mathbb{C}} \mathbb{S}^{n}\right)$, then the action of $\widehat{L}_{\mathbb{R}^{k} \times \mathbb{S}^{n}}^{*}(\zeta)$ is explicitly given by

$$
\begin{aligned}
{\left[\widehat{L}_{\mathbb{R}^{k} \times \mathbb{S}^{n}}^{*}(\zeta) \cdot \widehat{W}\right]^{\mathbb{C}^{k}=} } & -\frac{1}{2} \Delta_{\mathbb{S}^{n}} U+\left(\frac{m-2}{2 m}\right) \cdot\langle\zeta, U\rangle \cdot \zeta \\
& +\frac{1}{2} \cdot|\zeta|^{2} U-i\left(\frac{m-2}{2 m}\right) \cdot \operatorname{div}_{\mathbb{S}^{n}} V \cdot \zeta \\
{\left[\widehat{L}_{\mathbb{R}^{k} \times \mathbb{S}^{n}}^{*}(\zeta) \cdot \widehat{W}\right]^{T_{\mathbb{S}^{n}}=} } & L_{\mathbb{S}^{n}} V-\left(\frac{m-n}{m \cdot n}\right) \cdot \operatorname{grad}_{\mathbb{S}^{n}} \circ \operatorname{div}_{\mathbb{S}^{n}} V \\
& +\frac{1}{2}|\zeta|^{2} \cdot V-i\left(\frac{m-2}{2 m}\right) \cdot \operatorname{grad}_{\mathbb{S}^{n}}\langle\zeta, U\rangle
\end{aligned}
$$

For every $\zeta \in \mathbb{R}^{k}$ we associate to each $L_{\mathbb{R}^{k} \times \mathbb{S}^{n}}^{*}(\zeta)$ the adjoint operator

$$
\widehat{L}_{\mathbb{R}^{k} \times \mathbb{S}^{n}}(\zeta): H_{\gamma+n / 2}^{1} \rightarrow H_{\gamma+n / 2-2}^{1}
$$

It follows from the general theory of the elliptic operators acting between weighted spaces (see [13]) that $\widehat{L}_{\mathbb{R}^{k} \times \mathbb{S}^{n}}^{*}(\zeta)$ is surjective if and only if $\widehat{L}_{\mathbb{R}^{k} \times \mathbb{S}^{n}}(\zeta)$ is injective, $\zeta \in \mathbb{R}^{k}$.

Let now $Y \in H_{\gamma+n / 2}^{1}$ be a vector field such that $\widehat{L}_{\mathbb{R}^{k} \times \mathbb{S}^{n}}(\zeta) Y=0$. Since $\delta \in(2-n$, $(2-n) / 2)$, we can always choose $\gamma \in(\delta,(2-n) / 2)$. For $\gamma$ in this interval $H_{\gamma+n / 2}^{1} \subset H_{-\gamma-n / 2+2}^{1}$ and we can integrate by parts the expression

$$
0=\left(\widehat{L}_{\mathbb{R}^{k} \times \mathbb{S}^{n}}(\zeta) Y, Y\right)_{L^{2}}
$$


Writing $Y \in H_{\gamma+n / 2}^{1}$ as $Y=\Phi+\Psi$, with the decay prescriptions $\Phi \in H_{\gamma+n / 2}^{1}\left(\mathbb{S}^{n}, \mathbb{C}^{k}\right)$ and $\Psi \in H_{\gamma+n / 2}^{1}\left(\mathbb{S}^{n}, T^{\mathbb{C}^{n}}\right)$, we find

$$
\begin{aligned}
0= & \frac{1}{2} \int_{\mathbb{S}^{n}} \sum_{j=1}^{k}\left|\operatorname{grad}_{\mathbb{S}^{n}} \Phi^{j}\right|^{2} \text { dvol }_{\mathbb{S}^{n}}+\left(\frac{m-2}{2 m}\right) \int_{\mathbb{S}^{n}}|\langle\zeta, \Phi\rangle|^{2} \text { dvol }_{\mathbb{S}^{n}} \\
& +\frac{|\zeta|^{2}}{2} \int_{\mathbb{S}^{n}}|\Phi|^{2} \text { dvol }_{\mathbb{S}^{n}}+\frac{|\zeta|^{2}}{2} \int_{\mathbb{S}^{n}}|\Psi|^{2} \text { dvol }_{\mathbb{S}^{n}} \\
& +\int_{\mathbb{S}^{n}}\left|D_{\mathbb{S}^{n}} \Psi\right|^{2} \text { dvol }_{\mathbb{S}^{n}}+\left(\frac{m-n}{m \cdot n}\right) \int_{\mathbb{S}^{n}}\left|\operatorname{div}_{\mathbb{S}^{n}} \Psi\right|^{2} \text { dvol }_{\mathbb{S}^{n}} .
\end{aligned}
$$

Therefore, when $\zeta \neq 0$, we deduce at once that $\|\Phi\|_{L^{2}}^{2}=0=\|\Psi\|_{L^{2}}^{2}$, hence $Y=0$. When $\zeta=0$ we have that $\left\|\operatorname{grad}_{\mathbb{S}^{n}} \Phi^{j}\right\|_{L^{2}}^{2}=0$ for every $j=1, \ldots, k$, hence each component of $\Phi$ is a constant function and the decay prescription forces $\Phi$ to be zero. Concerning $\Psi$, we obtain the condition $\left\|D_{\mathbb{S}^{n}} \Psi\right\|_{L^{2}}^{2}=0$. This means that $\Psi$ is a conformal Killing vector field on $\mathbb{S}^{n}$, but it is well known that on an asymptotically Euclidean manifold there are no non trivial conformal Killing vector fields which decay at infinity [3], [10]. Hence $\Psi=0$ and $Y=0$ as well.

The injectivity of $\widehat{L}_{\mathbb{R}^{k} \times \mathbb{S}^{n}}(\zeta)$ for every $\zeta \in \mathbb{R}^{k}$ implies that the operator $L_{\mathbb{S}^{n} \times \mathbb{R}^{k}}^{*}$ is surjective. As a consequence, a solution $X$ to problem (35) must be identically zero, which is a contradiction.

\subsubsection{Case 3: the equation $L_{\mathbb{R}^{k} \times \mathbb{R}^{n}} X=0$ and the refined blow-up}

The analysis of the third case is very similar to the analysis of the second one, with the only substantial difference of the blow-up construction. Roughly speaking the slower velocity of the $p_{j}$ 's in tending to $p_{\infty}$ forces us to refine the blow-up procedure used in the previous section. In particular, if we want to control the behavior of the $p_{j}$ 's (which we need to do in order to carry out the argument by contradiction, since it guarantees the non-triviality of the solution), we need to choose their rate of approach to $p_{\infty}$ as rate of the blow-up of the other objects involved. If we set $\omega_{j}:=d_{g_{1}}\left(p_{j}, p_{\infty}\right)$, for $j \in \mathbb{N}$, we have that in this case $\varepsilon_{j} / \omega_{j} \rightarrow 0$ as $j \rightarrow 0$. Now, with the notation introduced in the previous section, it is quite natural to define a new family of diffeomorphisms $\left(\phi_{\omega_{j}}\right)_{j \in \mathbb{N}}$ via

$$
\begin{aligned}
\phi_{\omega_{j}}: B^{k}\left(1 / \omega_{j}\right) \times A^{n}\left(\varepsilon_{j} / \omega_{j}, 1 / \omega_{j}\right) & \longrightarrow B^{k}(1) \times A^{n}\left(\varepsilon_{j}, 1\right) \\
(z, x) & \longmapsto\left(\omega_{j} z, \omega_{j} x\right),
\end{aligned}
$$

and consequently define a family of new metrics $g_{\omega_{j}}^{\circ}$ as follows:

$$
\stackrel{\circ}{g_{\omega_{j}}}:=\frac{1}{\omega_{j}^{2}} \cdot\left[\phi_{\omega_{j}}^{*} g_{\varepsilon_{j}}\right] .
$$

In the limit $j \rightarrow+\infty$, the domain of definition of the $\phi_{\omega_{j}}$ 's becomes $\mathbb{R}^{k} \times\left(\mathbb{R}^{n} \backslash\{0\}\right)$ and the blow-up metrics $g_{\omega_{j}}^{\circ}$ tend to $g_{\mathbb{R}^{k}} \times g_{\mathbb{R}^{n}}$ on the compact sets of $\mathbb{R}^{k} \times\left(\mathbb{R}^{n} \backslash\{0\}\right)$ in the $\mathcal{C}^{2}$ 
topology. In fact the local expression for the $g_{\omega_{j}}^{\circ}$ reads

$$
\begin{aligned}
{\stackrel{\circ}{\omega_{j}}}^{\circ}(z, x)= & \left\{\delta_{i l}+\mathcal{O}\left(\omega_{j}|x|\right)+\mathcal{O}\left(\omega_{j}^{2}|z|^{2}\right)\right\} \cdot d z^{i} \otimes d z^{l} \\
& +\mathcal{O}\left(\omega_{j}|x|\right) \cdot d z^{i} \otimes d x^{\alpha} \\
& +\left[|x|^{\frac{n-2}{2}}+\left(\frac{\varepsilon_{j}}{\omega_{j}}\right)^{n-2}|x|^{-\frac{n-2}{2}}\right]^{\frac{4}{n-2}} \cdot\left\{|x|^{-2} \delta_{\alpha \beta}+\mathcal{O}\left(\omega_{j}^{2}\right)\right\} \cdot d x^{\alpha} \otimes d x^{\beta} .
\end{aligned}
$$

Proceeding as in the previous case we are led to the problem (36). In particular, we notice that the $p_{j}$ 's lie in a compact region of $\mathbb{R}^{k} \times S^{n-1}$. Hence, up to a subsequence, they converge to a point $p_{\infty}$. The rest of the analysis is very similar to the second case, since, mutatis mutandis, the decay conditions at infinity prescribed in (36) are the same as in (35). Rephrasing the argument of the previous subsection, we obtain that a solution to problem (36) must be identically zero, which is a contradiction.

This completes the proof of Proposition 2. As a consequence we immediately get an $\varepsilon$-uniform bound for the correction term $\sigma_{\varepsilon}$, and then for the TT-tensor $\mu_{\varepsilon}=\mu+\sigma_{\varepsilon}$, which solves the equations

$$
\begin{aligned}
\operatorname{tr}_{g_{\varepsilon}} \mu_{\varepsilon} & =0, \\
\operatorname{div}_{g_{\varepsilon}} \mu_{\varepsilon} & =0 .
\end{aligned}
$$

In the next section, we put $\mu_{\varepsilon}$ in the Lichnerowicz equation (for the metric $g_{\varepsilon}$ and the constant mean curvature $\tau$ ), and we use the $\varepsilon$-uniform bound of Proposition 2 to carry out a perturbation argument. By this method we will produce a solution $u_{\varepsilon}$ to the Lichnerowicz such that the closer $\varepsilon$ is to zero, the closer $u_{\varepsilon}$ is to one. As explained in the Introduction, this means that the new solution of the constraint approaches the starting ones, as $\varepsilon$ tends to zero.

\section{The energy constraint}

The aim of this section is to produce a solution to the $\varepsilon$-parameterized equation

$$
\Delta_{g_{\varepsilon}} u+c_{m} R_{g_{\varepsilon}} u-c_{m}\left|\mu_{\varepsilon}\right|_{g_{\varepsilon}}^{2} u^{-\frac{3 m-2}{m-2}}+c_{m} \frac{m-1}{m} \tau^{2} u^{\frac{m+2}{m-2}}=0,
$$

where $\mu_{\varepsilon}$ is the TT-tensor obtained in the Section 3. As claimed above, we also provide the solution with suitable estimates so that we have a control of the new Cauchy data set in terms of the old ones, as $\varepsilon$ tends to zero.

Our goal is achieved by means of a perturbation argument analogous to the one developed in [19]. Since the equation we are interested in is nonlinear, the first step consists in linearizing the Lichnerowicz operator around the constant one, this is reasonable since we want the solution to be as close as possible to the starting ones, as $\varepsilon$ approaches to zero. What we obtain is the linear operator

$$
\mathcal{L}_{g_{\varepsilon}}=\Delta_{g_{\varepsilon}}-\chi_{1} \cdot\left(\left|\mu_{1}\right|_{g_{1}}^{2}+\tau^{2} / m\right)-\chi_{2} \cdot\left(\left|\mu_{2}\right|_{g_{2}}^{2}+\tau^{2} / m\right),
$$

where $\chi_{1}$ and $\chi_{2}$ are the smooth cut-off functions defined in Section 2, and the error term

$$
\begin{aligned}
F_{\varepsilon}(v):= & c_{m}\left(R_{g_{1}}-R_{g_{\varepsilon}}\right) \chi_{1}-c_{m}\left(\left|\mu_{1}\right|_{g_{1}}^{2}-\left|\mu_{\varepsilon}\right|_{g_{\varepsilon}}^{2}\right) \chi_{1} \\
& +c_{m}\left(R_{g_{2}}-R_{g_{\varepsilon}}\right) \chi_{2}-c_{m}\left(\left|\mu_{2}\right|_{g_{2}}^{2}-\left|\mu_{\varepsilon}\right|_{g_{\varepsilon}}^{2}\right) \chi_{2}
\end{aligned}
$$




$$
\begin{aligned}
& +c_{m}\left(R_{g_{1}}-R_{g_{\varepsilon}}\right) \chi_{1} v+b_{m}\left(\left|\mu_{1}\right|_{g_{1}}^{2}-\left|\mu_{\varepsilon}\right|_{g_{\varepsilon}}^{2}\right) \chi_{1} v \\
& +c_{m}\left(R_{g_{2}}-R_{g_{\varepsilon}}\right) \chi_{2} v+b_{m}\left(\left|\mu_{2}\right|_{g_{2}}^{2}-\left|\mu_{\varepsilon}\right|_{g_{\varepsilon}}^{2}\right) \chi_{2} v \\
& +c_{m}\left|\mu_{\varepsilon}\right|_{g_{\varepsilon}}^{2} h(v)-c_{m} \frac{m-1}{m} \tau^{2} f(v),
\end{aligned}
$$

where $c_{m}=-(m-2) /[4(m-1)], b_{m}=c_{m} \cdot(3 m-2) /(m-2)$ and

$$
\begin{aligned}
& h(v)=\left[(1+v)^{-\frac{3 m-2}{m-2}}-1+\left(\frac{3 m-2}{m-2}\right) v\right] \\
& f(v)=\left[(1+v)^{\frac{m+2}{m-2}}-1-\left(\frac{m+2}{m-2}\right) v\right] .
\end{aligned}
$$

Hence both $h$ and $f$ are $\mathcal{O}\left(|v|^{2}\right)$.

The second step amounts to produce $\varepsilon$-uniform a priori estimate for solutions of the linear equation

$$
\mathcal{L}_{g_{\varepsilon}} v=w .
$$

Notice that since $g_{\varepsilon}$ tends to the metric $g_{i}$ in the $\mathcal{C}^{2}$-topology on the compact sets of $M_{i} \backslash K$, for $i=1,2$, then the coefficient of $\mathcal{L}_{g_{\varepsilon}}$ tend to the coefficients of the operators $\mathcal{L}_{g_{i}}$ defined in (15) on the compact sets of $M_{i} \backslash K, i=1,2$. Once this is done we seek a suitable estimate for the error term $F_{\varepsilon}(v)$. Having the a priori estimate and the estimate of the error term we can solve the equation

$$
\mathcal{L}_{g_{\varepsilon}} v=F_{\varepsilon}(v)
$$

by means of a fixed point argument.

As noted above, since the equation we want to solve is very similar to the Yamabe equation and since the linearized Lichnerowicz operators for the starting initial data set are injective (15), it is sufficient to adapt the argument used in [19] to our case. Let us focus, for instance, on the part of the error term $F_{\varepsilon}(v)$ which is supported on $M_{1}$ (the same is true for the other part of the error term). In order to apply successfully the Schauder fixed point theorem as in [19], it suffices that the "zero order" term in $F_{\varepsilon}(v)$ satisfies the estimate

$$
\left(R_{g_{1}}-R_{g_{\varepsilon}}\right)-\left(\left|\mu_{1}\right|_{g_{1}}^{2}-\left|\mu_{\varepsilon}\right|_{g_{\varepsilon}}^{2}\right) \leq C \cdot \varepsilon^{n-2} \rho_{\varepsilon}^{1-n},
$$

for some positive constant $C>0$. Concerning the piece $R_{g_{1}}-R_{g_{\varepsilon}}$ this is exactly the estimate of the scalar curvature obtained in [19]. Since $\mu_{\varepsilon}=\mu+\sigma_{\varepsilon}$, the other piece is dominated by $\left|\sigma_{\varepsilon}\right|_{g_{\varepsilon}}^{2}+\left|\mu-\mu_{1}\right|_{g_{\varepsilon}}^{2}$. Since $\left|\mu-\mu_{1}\right|_{g_{\varepsilon}}^{2}$ is zero outside the boundary of the polyneck and since it is clearly bounded in the middle, we can concentrate on the squared norm of $\sigma_{\varepsilon}=D_{g_{\varepsilon}} X$, where $L_{g_{\varepsilon}} X=\sharp \operatorname{div}_{g_{\varepsilon}} \mu$.

It follows from proposition (2) that there exists a constant $C_{0}>0$ independent of $\varepsilon$ such that

$$
\left|D_{g_{\varepsilon}} X\right|_{g_{\varepsilon}} \leq C_{0} \cdot{ }^{\varepsilon}\left\|\operatorname{div}_{g_{\varepsilon}} \mu\right\|_{\mathcal{C}_{\delta-2}^{1}} \cdot \rho_{\varepsilon}^{\delta-1}
$$

Since our aim is to get the bound $\left|D_{g_{\varepsilon}} X\right|^{2} \leq C \cdot \varepsilon^{n-2} \rho_{\varepsilon}^{1-n}$, it is sufficient to prove that

$$
{ }^{\varepsilon}\left\|\operatorname{div}_{g_{\varepsilon}} \mu\right\|_{\mathcal{C}_{\delta-2}^{1}} \leq C_{1} \cdot \varepsilon^{\frac{n-2}{2}} \rho_{\varepsilon}^{\frac{3-n}{2}-\delta},
$$


for some positive constant $C_{1}>0$. Since $2-n<\delta<(2-n) / 2$, this reduces to proving that

$$
\left\{\begin{array}{l}
\left|\operatorname{div}_{g_{\varepsilon}} \mu\right|_{g_{\varepsilon}} \leq C_{2} \cdot \varepsilon^{\frac{1}{2}-\delta} \cdot \rho_{\varepsilon}^{\delta-2} \\
\left|\nabla \operatorname{div}_{g_{\varepsilon}} \mu_{\varepsilon}\right|_{g_{\varepsilon}} \leq C_{3} \cdot \varepsilon^{\frac{1}{2}-\delta} \cdot \rho_{\varepsilon}^{\delta-3}
\end{array}\right.
$$

for some positive constants $C_{2}, C_{3}>0$. If we choose the constant $0<c<1$ which appears in the construction of $\mu$ in Section 2 to be $c=3 /(4-2 \delta)$, then both these conditions are satisfied and we get the desired bound for $\left|D_{g_{\varepsilon}} X\right|_{g_{\varepsilon}}^{2}$.

What remains to prove is the a priori estimate for solutions to the linearized problem $\mathcal{L}_{g_{\varepsilon}} v=w$. Following [19], we want to prove that for every $\gamma \in(2-n, 0)$ there exists a positive constant $C_{n, \gamma}>0$ and a real number $\varepsilon_{0} \in(0,1)$ such that for every $\varepsilon \in\left(0, \varepsilon_{0}\right)$, if $v, w \in \mathcal{C}^{0}\left(M_{\varepsilon}\right)$ are functions satisfying $\mathcal{L}_{g_{\varepsilon}} v=w$, then

$$
{ }^{\varepsilon}\|v\|_{\mathcal{C}_{\gamma}^{0}\left(M_{\varepsilon}\right)} \leq C \cdot{ }^{\varepsilon}\|w\|_{\mathcal{C}_{\gamma-2}^{0}\left(M_{\varepsilon}\right)} \cdot
$$

As shown in [19], this result can be achieved as a consequence of an analogous local a priori estimate. More precisely it is sufficient to prove that for every $\gamma \in(2-n, 0)$ there exist a real number $\alpha=\alpha(n, \gamma)>0$ and a positive constant $C_{n, \gamma}$ such that for all $\varepsilon \in\left(0, \mathrm{e}^{-\alpha}\right)$, if $v, w \in \mathcal{C}^{0}\left(M_{\varepsilon}\right)$ are functions verifying $\mathcal{L}_{g_{\varepsilon}} v=w$, then

$$
{ }^{\varepsilon}\|v\|_{\mathcal{C}_{\gamma}^{0}\left(T_{\alpha}^{\varepsilon}\right)} \leq C_{n, \gamma} \cdot{ }^{\varepsilon}\|w\|_{\mathcal{C}_{\gamma-2}^{0}\left(T_{\alpha}^{\varepsilon}\right)}+{ }^{\varepsilon}\|v\|_{\mathcal{C}_{\gamma}^{0}\left(\partial T_{\alpha}^{\varepsilon}\right)},
$$

where $T_{\alpha}^{\varepsilon}$ is the portion of the poly-neck where $\alpha+\log \varepsilon \leq t \leq-\alpha-\log \varepsilon$. Since in [19] an analog of the estimate (76) is obtained for solutions to the equation $\Delta_{g_{\varepsilon}} v=w$, it is sufficient to adapt it to our case. Since for $\alpha$ large enough and $\varepsilon$ sufficiently small we have that

$$
\begin{gathered}
\left.\sup _{T_{\alpha}^{\varepsilon}}\left|\rho_{\varepsilon}^{-\gamma+2}\right| \mu_{1}\right|_{g_{1}} ^{2} v\left|\leq \frac{1}{4} \sup _{T_{\alpha}^{\varepsilon}}\right| \rho_{\varepsilon}^{-\gamma} v \mid \\
\sup _{T_{\alpha}^{\varepsilon}}\left|\rho_{\varepsilon}^{-\gamma+2}\left(\tau^{2} / m\right) v\right| \leq \frac{1}{4} \sup _{T_{\alpha}^{\varepsilon}}\left|\rho_{\varepsilon}^{-\gamma} v\right|,
\end{gathered}
$$

this implies (76). Having obtained all the estimates required, it is now possible to apply the Schauder fixed point theorem as in [19], provided that $\max \{-1,(2-n) / 2\}<\gamma<0$. Hence we find a solution $v_{\varepsilon}$ to the equation (70). Moreover we have that $\left\|v_{\varepsilon}\right\|_{L^{\infty}(M)}=\mathcal{O}\left(\varepsilon^{-\gamma}\right)$ and this completes the proof of Theorem 1.

\section{Conclusions and further directions}

The result provided in Theorem 1 allows one to build a new zoo of solutions to the vacuum Einstein equation, hence it provides a good instrument to investigate the structure of the space-time. Notice that in the case of the classical $(3+1)$-dimensional space-time, our result reduces to the IMP gluing, because of the hypothesis on the codimension of $K$. However people who study string theory, might find our result of some interest on the physical point of view.

Following [10] our result can be extended to the case of Asymptotically Euclidean (AE) and Asymptotically Hyperbolic ( $\mathrm{AH}$ ) initial data set without difficulty. In fact to adapt the proof, it is sufficient to slightly modify the functional setting, in order to guarantee the existence of an inverse for the vector Laplacian and for the linearized Lichnerowicz operator. 
Another possible improvement of our result consists in localizing the construction in order to produce a new initial data set which is exactly like the starting ones out of the polyneck, as it has already been done for the IMP gluing [5].

Finally (ongoing work) the structure of the polyneck should be further investigated. In particular we expect that in certain cases it is possible to find an apparent horizon in the middle of the polyneck, hence the space-time development of such initial data sets is forced to contain multidimensional black holes with possibly non trivial topology.

Acknowledgments The author would like to thank J. Isenberg, R. Mazzeo and D. Pollack for many helpful suggestions and discussions during the preparation of this paper.

Open Access This article is distributed under the terms of the Creative Commons Attribution Noncommercial License which permits any noncommercial use, distribution, and reproduction in any medium, provided the original author(s) and source are credited.

\section{References}

1. Aubin, T.: Some nonlinear problems in Riemannian Geometry, Springer Monographs in Mathematics. Springer, Heidelberg (1998)

2. Bartnik, R., Isenberg, J. : The constraint equations. In: Chrusciel, P.T., Friedrich, H. (eds.) The Einstein equations and the large scale behavior of gravitational fields, pp. 1-39. Birkhäuser, Basel (2004)

3. Christodoulou, D., Choquet-Bruhat, Y.: Elliptic systems in $H_{s, \delta}$ spaces on manifolds which are Euclidean at infinity. Acta Math. 146, 129-150 (1981)

4. Choquet-Bruhat, Y.: Théorème d'existence pour certains systèmes d'équations aux dérivées partielles non linéaires. Acta Math. 88, 141-225 (1952)

5. Chruściel, P.T., Isenberg, J., Pollack, D.: Initial data engineering. Comm. Math. Phys. 257, 29-42 (2005)

6. Giaquinta, M., Martinazzi, L.: An introduction to the regularity thoery for elliptic systems, harmonic maps and minimal graphs. Edizioni della Normale (2005)

7. Gilbarg, D., Trudinger N.: Elliptic partial differential equation of second order. Springer, Heidelberg (1983)

8. Gromov, M., Lawson, H.B.: The classification of simply connected manifolds of positive scalar curvature. Ann. Math. (2) 111(3), 423-434 (1980)

9. Joyce, D.: Constant scalar curvature metrics on connected sums. Int. J. Math. Math. Sci. 7, 405-450 (2003)

10. Isenberg, J., Mazzeo, R., Pollack, D.: Gluing and wormholes for the Einstein constraint equations. Comm. Math. Phys. 231, 529-568 (2002)

11. Isenberg, J., Maxwell, D., Pollack, D.: A gluing construction for non vacuum solutions of the Einstein constraint equations. Adv. Theor. Phys. 9, 129-172 (2005)

12. Lee, J.M., Parker, T.H.: The Yamabe Problem. Bull. AMS. 17, 37-91 (1987)

13. Mazzeo, R.: Elliptic theory of differential edge operators. Commun. Partial Differ. Equ. 16, 1615-1664 (1991)

14. Mazzeo, R., Pollack, D., Uhlenbeck, K.: Connected sums constructions for constant scalar curvature metrics. Topol. Method Nonlinear Anal. 6, 207-233 (1995)

15. Mazzeo, R., Pacard, F.: Constant scalar curvature metrics with isolated singularities. Duke Math. J. 99(3), 353-418 (1999)

16. Mazzeo, R., Pacard, F.: Constant mean curvature surfaces with Delaunay ends. Comm. Anal. Geom. 9(1), 169-237 (2001)

17. Mazzeo, R., Pacard, F., Pollack, D.: Connected sums of constant mean curvature surfaces in Euclidean 3 space. J. Reine Angew. Math. 536, 115-165 (2001)

18. Mazzieri, L.: Generalized connected sum construction for scalar flat metrics. arXiv:math.DG/0611778 (2006)

19. Mazzieri, L.: Generalized connected sum construction for nonzero constant scalar curvature metrics. Commun. Partial Differ. Equ. 33, 1-17 (2008)

20. Schoen, R., Yau, S.T.: On the structure of manifolds with positive scalar curvature. Manuscripta Math. 28(1-3), 159-183 (1979)

21. Taylor, M.: Partial differential equations III: nonlinear equations. Appl. Math. Sci. 117, Springer, New York (1996) 